Año 9, número 16, marzo - agosto 2019

Paakat: Revista de Tecnología y Sociedad

e-ISSN: 2007-3607

Universidad de Guadalajara

Sistema de Universidad Virtual

México

suv.paakat@redudg.udg.mx

Año 9, número 16, marzo - agosto 2019

\title{
Recensión \\ Ruidos en la web. Cómo se informan los adolescentes en la era digital
}

\author{
Joaquín Linne* \\ https://orcid.org/0000-0002-4297-3914
}

Consejo Nacional de Investigaciones Científicas y Técnicas (CONICET), Buenos Aires

Obra reseñada: Roxana Morduchowicz, 2018

Ruidos en la web. Cómo se informan los adolescentes en la era digital

Ediciones B, Buenos Aires, Argentina, 192 pp.

[Recibido 14/09/2018. Aceptado para su publicación 3/12/2018]

DOI: http://dx.doi.org/10.32870/Pk.a9n16.378

La investigadora argentina Roxana Morduchowicz es doctora en Comunicación por la Universidad de París, consultora de la UNESCO en temas de educación y tecnologías y profesora de Comunicación en la Universidad de Buenos Aires. Ha escrito diversos libros: Los adolescentes del siglo XXI (2013), Los chicos y las pantallas (2014) y La generación multimedia (2008), entre otros. En ellos se dedicó a describir y analizar las relaciones que los "nativos digitales" o "millenials" establecen con internet y con las plataformas de redes sociales. 
En el último libro se centra en el problema de las noticias falsas o fake news, asociado al ansioso y "acrítico" acceso a la información que realizan los jóvenes. Pero este problema los excede, ya que afecta a una significativa parte de los internautas:

En este mar de textos y discursos la navegación es confusa. Las olas de información desordenan y descontextualizan los saberes y vuelven más difícil reconocer qué textos utilizar, cuáles son relevantes y qué fuentes hablan con autoridad sobre un tema determinado. La abundancia de información, propia de la era digital, profundizó la desorientación para seleccionar contenidos de fuentes confiables (Morduchowicz, 2018, p. 12).

Al haber coordinado durante varios años el programa Escuela $y$ medios, dependiente del Ministerio de Educación de Argentina, diseñó e implementó encuestas a adolescentes sobre consumos culturales y usos de internet, lo cual después analizó.

En sus libros muestra una discusión bibliográfica actualizada, si bien más centrada en textos internacionales que locales y en encuestas más que en papers y artículos. En el caso de su reciente libro dialoga con autores de la talla de Danah Boyd, quien estudia el uso de sitios de redes sociales de adolescentes en Estados Unidos de América; Sonia Livingstone, quien investiga el uso de internet de niños y adolescentes en Europa; Jenkins, cuya propuesta en sus ensayos está dirigida hacia una cultura de la convergencia digital al tender a superponer usos y dispositivos en aquello que se denomina multitasking o realización de tareas múltiples, característica inicialmente más propia de las generaciones jóvenes.

En Ruidos en la web. Cómo se informan los adolescentes en la era digital, Morduchowicz aporta evidencia empírica y teórica sobre este problema de falta de sentido crítico que comparten la mayoría de adolescentes respecto de los contenidos de la web. En este sentido, señala que si bien siete de diez adolescentes argentinos están todo el día conectados a la web, la mayoría no adopta una mirada crítica sobre este conjunto de dispositivos y plataformas digitales, ni se interroga sobre su origen y financiamiento.

La mayoría tiende a quedarse con el primer sitio web encontrado al buscar una información sin detenerse a verificar la información ni a comparar distintas fuentes. Además, la sobrevaloración que realizan los internautas sobre la rapidez en el acceso a contenidos conlleva un significativo riesgo para distinguir las fuentes fiables de aquellas que no son, más allá de la dificultad contemporánea para darse el tiempo de procesar y reflexionar sobre la información leída.

Otro de los aspectos más relevantes del libro gira en torno a la problematización de la categoría nativos e inmigrantes digitales (Prensky, 2001). ¿Todos los jóvenes son "nativos digitales"? Distintos estudios cuestionan el alcance homogeneizador de esta categoría, dado que no establece diferencias entre sectores sociales ni por género ni por habitar en distintas geografías. Al mismo tiempo, el uso de esta categoría tiende a idealizar a los jóvenes como expertos digitales:

Me interesa hablar de los adolescentes para desafiar la expresión de "nativo digital". Este concepto, muy extendido en todo el mundo, supone que los chicos son 
nativos digitales porque nacieron con las tecnologías. Pone el énfasis en la edad, pero no dice nada de las competencias y las capacidades, que son las que, en realidad, definen cuándo un chico es un nativo digital. Si un adolescente no sabe diferenciar fuentes confiables de las que no lo son, o si "copia y pega" información sin analizarla, no puede recibir el nombre de "nativo digital", aunque haya nacido en el 2000. Podrá contar con un saber instrumental que le permita manejar la herramienta mucho mejor que sus padres, pero está lejos de ser un verdadero "nativo digital", que sólo puede ser definido como tal cuando es capaz de utilizar todo el potencial de Internet de manera reflexiva y creativa. Lo único que hace el concepto de "nativo digital" es desresponsabilizar a los adultos de la formación de los chicos (Morduchowicz, 2018, p. 15).

Pero Morduchowicz no es la única que critica este difundido concepto. Por ejemplo, en su libro Los nativos digitales no existen los españoles Lluna y Pedreira (2017) sostienen:

Estamos acostumbrados a oír la expresión "nativo digital" para referirse a los nacidos a partir de mediados de los noventa, quienes, supuestamente acostumbrados a la presencia de ordenadores y otros dispositivos digitales en sus vidas, no necesitan que nadie les enseñe a utilizarlos. [...] Considerar que estos jóvenes van a saber aprovechar el enorme potencial de estas tecnologías en su desarrollo como personas y en el progreso de nuestra sociedad de forma casi instintiva, sin que tengan el apoyo de la familia y sin que diseñemos y apliquemos planes educativos al respecto, resulta absurdo.

Recordemos que el polémico término es más bien un binomio (nativos digitales/inmigrantes digitales), el cual divide a la sociedad a partir de su facilidad para utilizar las tecnologías de la información y la comunicación (TIC), dependiendo de si pasaron miles de horas con sitios de redes sociales, páginas web, videojuegos y chats, 0 no. Por una cuestión de "moratoria social" (Margulis y Urresti, 1998), es decir, de disponibilidad temporal, una gran parte de los jóvenes tienen o han tenido mucho más tiempo que los adultos para entrenarse y socializarse en estas nuevas tecnologías.

También es cierto que niños, adolescentes y jóvenes de sectores populares, al disponer de una menor moratoria social, suelen poseer menos tiempo para volverse "usuarios expertos" en estas tecnologías. En todo caso, la autora señala que la mayoría de los adolescentes, más allá de que sean nativos digitales para usos instrumentales, lúdicos y comunicativos, a nivel informativo suelen ser "ingenuos digitales".

En definitiva, Ruidos en la web alerta sobre la transversalidad de estos problemas: cada vez son más quienes se informan principalmente a través de redes sociales, no solo son los jóvenes. Al mismo tiempo señala la necesidad de aggiornar las instituciones educativas para incorporar los lenguajes digitales. En particular, recomienda enseñar y entrenar a las nuevas generaciones para que puedan leer críticamente distintos formatos, tanto los tradicionales de los libros, diarios y revistas, como los nuevos formatos propios de la web:

La escuela necesita formar un alumno que sepa pensar qué lugar ocupa la información en su vida, que comprenda de qué manera incide en su percepción del

Paakat: Revista de Tecnología y Sociedad

Año 9, núm. 16, marzo-agosto 2019, e-ISSN: 2007-3607 
mundo y que aprenda cómo utilizarla, para exigir la mejor calidad informativa y para tomar las mejores decisiones. En definitiva, para que, frente a la tarea escolar, el objetivo del estudiante no sea "copiar y pegar" el primer link que aparece en el buscador (Morduchowicz, 2018, p. 160).

Para esto plantea que las escuelas podrían cambiar las consignas que les dan a los estudiantes, saliendo del paradigma memorístico para buscar tareas que les resulten más desafiantes. De este modo, se podría contribuir a reducir la brecha entre las prácticas que realizan dentro de la escuela y aquellas que hacen afuera, ampliando la utilidad de la escuela para alfabetizar a los estudiantes en competencias digitales novedosas como seleccionar información y recursos de la web de maneras más expertas, creativas y críticas.

\section{Referencias}

Lluna, S. y Pedreira, J. (2017). Los nativos digitales no existen. Barcelona, España: Centro Libros PAPF.

Margulis, M. y Urresti, M. (1998). La construcción social de la juventud. Revista Question. Recuperado de http://www.perio.unlp.edu.ar/catedras/system/files/mario_margulis_y_marcelo_ urresti_-_la_construccion_social_de_la_condicion_de_juventud_urresti.pdf

Morduchowicz, R. (2018). Ruidos en la web. Cómo se informan los adolescentes en la era digital. Buenos Aires, Argentina: Ediciones B.

Prensky, M. (2001). Digital Natives, Digital Inmigrants. On the Horizon, 1 (5), 1-6. Recuperado de https://www.marcprensky.com/writing/Prensky\%20\%20 Digital\%20Natives,\%20Digital\%20Immigrants\%20-\%20Part1.pdf

\footnotetext{
* Joaquín Linne. Doctor en Ciencias Sociales, Magíster en Comunicación y Cultura y Lic. en Sociología por la UBA (graduado con honores). Investigador Asistente del CONICET con sede de trabajo en el Instituto Gino Germani. Ha publicado 25 artículos de investigación en revistas con referato internacional indexadas en Scopus, Scielo, Redalyc y Latindex. Además de reseñas, columnas, ponencias y capítulos de libros, es coautor de un libro de divulgación científica (Conexión total. Los jóvenes y la experiencia social en la era de la comunicación digital, 2015) y uno de crónicas (Misoginia latina, 2010). Participó como expositor, comentarista y coorganizador en 40 reuniones científicas. Fue evaluador externo en 40 artículos como parte del sistema doble ciego de referato. En la actualidad, es docente en UBA y UNLa. Además, codirige y participa de grupos de investigación en UNLa, UBA y Flacso/UNIPE. Desde allí, continúa investigando sobre problemáticas juveniles vinculadas al género, la educación y las culturas digitales.
} 